\title{
Magnetic Resonance Sentinel Lymph Node Imaging and Magnetometer-Guided Intraoperative Detection in Penile Cancer, using Superparamagnetic Iron Oxide Nanoparticles: First Results
}

\author{
Alexander Winter ${ }^{\mathrm{a}}$ Tobias Kowald $^{\mathrm{b}}$ Svenja Engels ${ }^{\mathrm{a}} \quad$ Friedhelm Wawroschek $^{\mathrm{a}}$ \\ a University Hospital for Urology, Klinikum Oldenburg, School of Medicine and Health Sciences, Carl von Ossietzky \\ University Oldenburg, Oldenburg, Germany; ${ }^{b}$ Institute of Diagnostic and Interventional Radiology, Klinikum Oldenburg, \\ Oldenburg, Germany
}

\section{Keywords}

Penile cancer · Sentinel lymphadenectomy .

Magnetometer - Magnetic resonance imaging .

Superparamagnetic iron oxide nanoparticles

\begin{abstract}
In penile cancer, lymph node (LN) metastasis is the main known prognostic factor that affects survival. Inguinal sentinel LN (SLN) dissection (SLND) using radioactive marking is recommended by the European Association of Urology guidelines to evaluate the nodal status in clinically nodenegative penile cancer ( $c N 0 ; \geq p T 1, G 2$ ). Dependence on radioisotopes limits the application of this procedure to small parts of the developed world, and imposes restrictions on hospital logistics. To overcome these issues, SLN visualization using magnetic resonance imaging (MRI) and magnetometer-guided detection after intraprostatic injection of superparamagnetic iron oxide nanoparticles (SPION) has been successfully applied in prostate cancer. Here, we present the first results of magnetic sLND in penile cancer. After peritumoral SPION injection, MR SLN imaging and magnetometer-guided SLND were performed in one cNO penile cancer patient. Another patient underwent magnetometerguided sLND only. In the first case, $5 \mathrm{SLN}$ s could be visualized on MRI and intraoperatively detected by magnetometer-
\end{abstract}

karger@karger.com

(c) 2019 S. Karger AG, Basel

www.karger.com/uin

Karger" guided sLND. In the second patient, 3 SLNs could be detected by magnetic sLND. Neither patient exhibited adverse events attributable to SPION-injection. In conclusion, SPIONguided SLN identification using MRI and a handheld magnetometer is feasible and could provide a radiation-free technique for SLN identification in penile cancer. For further clarification, a multicenter study should be carried out.

(c) 2019 S. Karger AG, Base

\section{Introduction}

Lymph node (LN) staging in penile cancer has strong prognostic implications. Early inguinal LN dissection (LND) or resection of clinically occult LN metastases improves survival compared with removal after metastases become clinically apparent [1]. LND can detect micrometastatic LN involvement which is undetectable by current imaging modalities. However, the benefits of radical inguinal LND are tempered by high morbidity or over-treatment of many patients $[2,3]$. To reduce morbidity associated with radical inguinal LND, the European Association of Urology (EAU) guidelines recommend radio-guided sentinel LND (sLND) using peritumoral injection of ${ }^{99} \mathrm{~m}_{\text {technetium- }}$ nanocolloid, preoperative scintigraphy, and a gammaprobe for intraoperative detection or modified inguinal 
Table 1. Parameters of the SPION-MRI sequences obtained after peritumoral SPION injection for identification of sentinel lymph nodes in one clinical node negative $(\mathrm{cN} 0)$ penile cancer patient

\begin{tabular}{|c|c|c|c|c|c|c|}
\hline Parameters & \multicolumn{6}{|c|}{ Sequences } \\
\hline TR/TE, ms & $5,350 / 67$ & $659 / 10$ & $3,500 / 89$ & $3,500 / 89$ & $\begin{array}{l}40 / 1.26 \\
40 / 2.86\end{array}$ & $2,734 / 61$ \\
\hline Flip angle, ${ }^{\circ}$ & 150 & 141 & 160 & 160 & 20 & $\mathrm{~N} / \mathrm{A}$ \\
\hline Matrix & 256 & 256 & 320 & 320 & 208 & 128 \\
\hline Imaging time, min & $2: 31$ & $2: 48$ & $2: 58$ & $2: 40$ & $3: 14$ & $1: 56$ \\
\hline
\end{tabular}

SPION, superparamagnetic iron oxide nanoparticles; MRI, magnetic resonance imaging; STIR, short-tau inversion recovery; SE, spin echo; TSE, turbo; GRE, gradient-recalled echo; DW, diffusion weighted; EPI, echo planar imaging; TR, repetition time; TE, echo time; FOV, field of view; N/A, not applicable.

LND for clinically node-negative ( $\mathrm{cN} 0)$ penile cancer patients ( $\geq \mathrm{pT} 1, \mathrm{G} 2)$ [4]. Nevertheless, these radioactive sentinel LN (SLN) procedures have some drawbacks (e.g., dependence on nuclear medicine facilities). To overcome these limitations, superparamagnetic iron oxide nanoparticles (SPION) have been successfully applied to identify SLNs in breast and prostate cancers $[5,6]$. Here, we report the first results of magnetic SLN identification using MR SLN imaging and a handheld magnetometer after peritumoral SPION injection in 2 patients with $\mathrm{cN} 0$ penile cancer.

\section{Case Report/Case Presentation}

Two patients with cN0 penile cancer underwent magnetic inguinal sLND after being referred to our university hospital for sLND combined with partial penile resections. The first patient (aged 64 years) had a histologically confirmed penile cancer at the glans. The second patient (aged 43 years) presented with a penile cancer of the foreskin. Radioactive labeling of SLNs was not possible for these patients as ${ }^{99 \mathrm{~m}}$ technetium tracer and nuclear medicine facilities were unavailable. Therefore, they underwent magnetic marking and intraoperative detection of SLNs, using a SPI$\mathrm{ON}$ tracer and a handheld magnetometer.

The SPION tracer was a component of the SentiMag ${ }^{\circledR}$ system (Endomagnetics Ltd., Cambridge, UK). This system for marking and identifying SLNs comprised a handheld magnetometer (SentiMag ${ }^{\circledR}$ ) and the Sienna $+{ }^{\circledR}$ magnetic tracer. Sienna $+{ }^{\circledR}$ is classified as a class IIa medical device by Medical Device Directive 93/42/ EEC. The particles have a carboxydextran coating and a mean hydrodynamic diameter of $60 \mathrm{~nm}$. Sienna $+{ }^{\circledR}$ has functional properties comparable to ${ }^{99 \mathrm{~m}}$ technetium nanocolloid. As with the radionuclide, the tracer flows through the lymph system and gets trapped in the SLNs after interstitial injection.

In both patients, the SPION tracer $(1 \mathrm{~mL})$ was injected and evenly spread as several peritumoral deposits $3 \mathrm{~h}$ before surgery. The first patient underwent magnetic resonance imaging (MRI) for preoperative visualization of SLNs. The MRI study was conducted using a 1.5-T MRI Scanner (MAGNETOM Aera; Siemens, Erlangen, Germany) as previously described $[7,8]$. The acquisition of the MR images started one and a half hour after injection. We performed $\mathrm{T} 2 *$-weighted gradient echo scans in the transversal plane to localize the LNs with SPION uptake (Table 1). In the second patient, MRI was preoperatively not available. As well as planar lymphoscintigraphy or single-photon emission CT in the radioactive approach, preoperative MRI can provide the surgeon with a road map for magnetic intraoperative SLN detection. However, performing MRI is not mandatory before magnetometer-guided sentinel identification. Both patients underwent magnetometer-guided sLND and partial penectomy or resection of the foreskin.

In the first patient, 3 SLNs were preoperatively visualized in the right groin and 2 SLNs in the left groin (Fig. 1). Intraoperatively, all SLNs could be detected using the handheld magnetometer, leading to a diagnosis of $\mathrm{pT} 2 \mathrm{pN} 0$ (sn; 0/5)/L0 V0 G2. In the second patient, 3 histopathological negative SLNs were intraoperatively detected by magnetic sLND and 1 adherent non-SLN was additionally resected leading to a diagnosis of pTla pN0 (sn; 0/4)/L0 V0 G2. Both patients are currently relapse-free at 39 and 5 months, respectively.

\section{Discussion}

Because of high morbidity from extended inguinal LND or over-treatment of many patients, inguinal-modified LND or SLND is recommended by the EAU guidelines to evaluate the nodal status of $\mathrm{cN} 0$ penile cancer patients. Excellent results concerning sensitivity, false-negative rates, and complication rates have been reported for radio-guided sLND in experienced hands [9-11]. However, dependence on radioisotopes limits this procedure to small parts of the developed world, and imposes restrictions on patient planning and hospital logistics. It also exposes patients and surgical staff to radiation. How- 


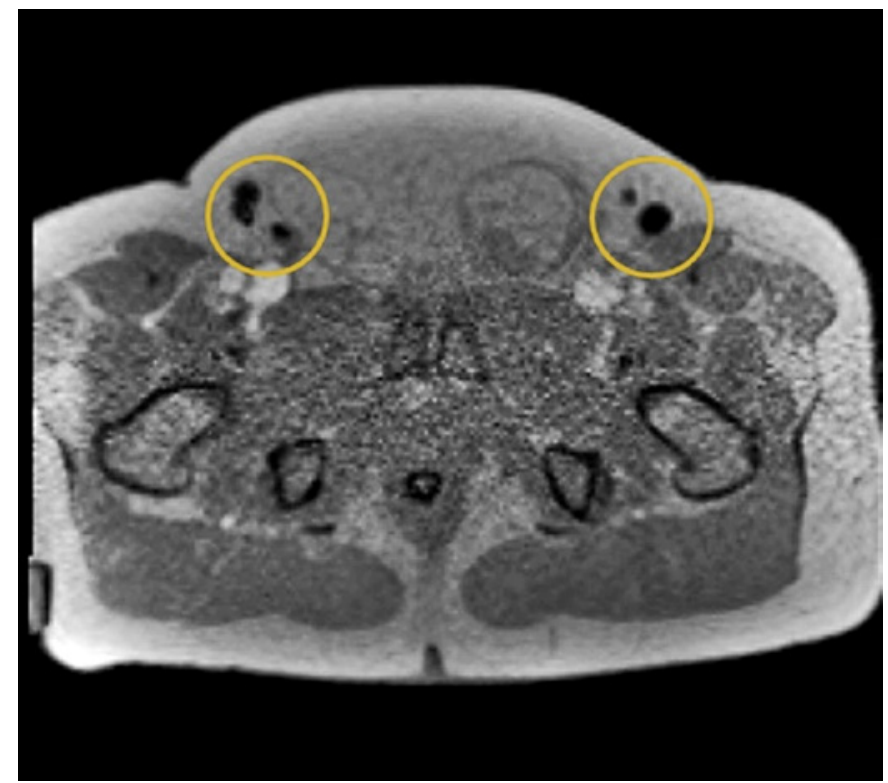

Fig. 1. After peritumoral administration of superparamagnetic iron oxide nanoparticles, 3 SLNs in the right groin and 2 SLNs in the left groin showed a strong drop in signal intensity on postcontrast $\mathrm{T} 2 *$-weighted magnetic resonance image.

ever, the exposure to radiation is relatively low (patient $<0.5 \mathrm{mSv}$; surgeon ca. $0.2-3.7 \mu \mathrm{Sv}$ per patient). Therefore, surgeons do not have to be classified as occupationally exposed persons, even if they frequently perform the radioactive sentinel procedure. This would only be required if an annual dose of $1,000 \mu \mathrm{Sv}$ would be exceeded. To overcome these impediments, SPION have been successfully used to identify SLNs in breast cancer [5]. Recently, we presented the first results of preoperative visualization and intraoperative detection of SLNs in prostate cancer, using a magnetic tracer, MRI, and a handheld magnetometer $[7,8]$. Because of the unavailability of ${ }^{99} \mathrm{~m}_{\text {technetium }}$ or nuclear medicine facilities, we used this magnetic sentinel approach in 2 patients with $\mathrm{cN} 0$ penile cancer.

To our knowledge, this is the first report on the use of magnetic marking and magnetometer-guided SLN detection in penile cancer. We were able to show that magnetic identification of SLNs using a SPION tracer and a handheld magnetometer is suitable for penile cancer patients. Although these 2 cases were the first times that this procedure was ever used for penile cancers, we could intraoperatively detect an adequate number of SLNs in each case.

Use of MRI after peritumoral SPION injection to preoperatively localize SLNs in penile cancer has not yet been reported. In 1 patient who underwent preoperative MRI, we showed that magnetic visualization of SLNs under

Magnetic Sentinel Node Detection in Penile Cancer
MRI is feasible; this technique allowed reliable identification of all intraoperatively detected SLNs. Therefore, MR SLN imaging can provide surgeons with important preoperative information on the location of magnetically marked SLNs or a road map for magnetometer-guided intraoperative SLN detection. If possible, preoperative SLN imaging should be carried out.

\section{Conclusion}

To reduce morbidity associated with radical inguinal LND, the EAU guidelines recommend radio-guided sLND for patients with $\mathrm{cN} 0$ penile cancer ( $\geq \mathrm{pT} 1, \mathrm{G} 2)$. However, radio-guided sLND has some drawbacks due to the radioactive marking. In the present report of 2 patients with penile cancer, for the first time, SLNs were reliably detected using a SPION tracer and a handheld magnetometer. This case study also shows that SLNs can be visualized by MRI after peritumoral SPION injection in penile cancer. Initial data indicate that this simple, radiation-free magnetic procedure is safe and feasible, and provides an entirely radiationfree technique for pre- and intraoperative SLN localization in penile cancer. Should these initial findings be confirmed in further studies, magnetic SLN identification offers the potential for targeted LN surgery in penile cancer. Based on these feasibility data, a multicenter study including intraindividual comparison of ${ }^{99} \mathrm{~m}_{\text {technetium nanocolloid and }}$ SPION should be started for further clarification.

\section{Acknowledgement}

We thank Marla Brunker, from Edanz Group (www.edanzediting.com/ac), for editing a draft of this manuscript.

\section{Statement of Ethics}

The 2 subjects have given their written informed consent to publish their case including publication of images. The study complies with the guidelines for human studies and was conducted in accordance with the World Medical Association Declaration of Helsinki.

\section{Disclosure Statement}

The authors have no conflicts of interest to declare.

\section{Funding Sources}

None. 


\section{References}

1 Kroon BK, Horenblas S, Lont AP, Tanis PJ, Gallee MP, Nieweg OE. Patients with penile carcinoma benefit from immediate resection of clinically occult lymph node metastases. J Urol. 2005 Mar;173(3):816-9.

2 Bevan-Thomas R, Slaton JW, Pettaway CA Contemporary morbidity from lymphadenectomy for penile squamous cell carcinoma: the M.D. Anderson Cancer Center Experience. J Urol. 2002 Apr;167(4):1638-42.

3 Protzel C, Alcaraz A, Horenblas S, Pizzocaro G, Zlotta A, Hakenberg OW. Lymphadenectomy in the surgical management of penile cancer. Eur Urol. 2009 May;55(5):1075-88.

4 Hakenberg OW, Compérat EM, Minhas S, Necchi A, Protzel C, Watkin N. Guidelines Associate: Robinson R. EAU Guidelines on Penile Cancer (Accessed March 24, 2019). Available from: http://www.uroweb.org/ guideline/penile-cancer/.

5 Douek M, Klaase J, Monypenny I, Kothari A, Zechmeister K, Brown D, et al.; SentiMAG
Trialists Group. Sentinel node biopsy using a magnetic tracer versus standard technique: the SentiMAG Multicentre Trial. Ann Surg Oncol. 2014 Apr;21(4):1237-45.

6 Winter A, Woenkhaus J, Wawroschek F. A novel method for intraoperative sentinel lymph node detection in prostate cancer patients using superparamagnetic iron oxide nanoparticles and a handheld magnetometer: the initial clinical experience. Ann Surg Oncol. 2014 Dec;21(13):4390-6.

7 Winter A, Chavan A, Wawroschek F. Magnetic resonance imaging of sentinel lymph nodes using intraprostatic injection of superparamagnetic iron oxide nanoparticles in prostate cancer patients: first-in-human results. Eur Urol. 2018 May;73(5):8134.

8 Winter A, Kowald T, Paulo TS, Goos P, Engels $\mathrm{S}$, Gerullis $\mathrm{H}$, et al. Magnetic resonance sentinel lymph node imaging and magnetometer-guided intraoperative detection in pros- tate cancer using superparamagnetic iron oxide nanoparticles. Int J Nanomedicine. 2018 Oct;13:6689-98.

9 Lam W, Alnajjar HM, La-Touche S, Perry M, Sharma D, Corbishley C, et al. Dynamic sentinel lymph node biopsy in patients with invasive squamous cell carcinoma of the penis: a prospective study of the long-term outcome of 500 inguinal basins assessed at a single institution. Eur Urol. 2013 Apr;63(4):657-63.

10 Schubert T, Uphoff J, Henke RP, Wawroschek F, Winter A. Reliability of radioisotopeguided sentinel lymph node biopsy in penile cancer: verification in consideration of the European guidelines. BMC Urol. 2015;28:15: 98.

11 Sadeghi R, Gholami H, Zakavi SR, Kakhki VR, Tabasi KT, Horenblas S. Accuracy of sentinel lymph node biopsy for inguinal lymph node staging of penile squamous cell carcinoma: systematic review and meta-analysis of the literature. J Urol. 2012 Jan;187(1):25-31. 